\title{
Aproximación sociopolítica para el análisis de políticas de conservación en contextos urbanos: entre servicios ambientales y áreas naturales protegidas \\ Sociopolitical approach for the analysis of conservation policies in urban contexts: Between environmental services and protected natural areas
}

\author{
Gabriela De la Mora-De la Mora*
}

Perfiles Latinoamericanos, 27(53) | 2019

DOI: $10.18504 / \mathrm{pl} 2753-003-2019$

Recibido: 28 de enero de 2017

Aceptado: 28 de abril de 2018

\begin{abstract}
Resumen
En este artículo se propone una aproximación sociopolítica para analizar los retos que enfrentan los tomadores de decisiones y la sociedad en general respecto de la implementación de dos políticas de conservación convergentes en contextos urbanos en México: los servicios ambientales o ecosistémicos hidrológicos y las áreas naturales protegidas. Se delinean teórica y metodológicamente los componentes de una aproximación sociopolítica para analizar ambas políticas de conservación en la literatura académica de reciente publicación. Se estudian ambos instrumentos de política pública y los procesos sociales e interacciones que desatan con su puesta en marcha para lograr la conservación de socioecosistemas en contextos urbanos en México. Se concluye que es indispensable estudiar estos temas con mayor profundidad.
\end{abstract}

\begin{abstract}
This paper proposes a sociopolitical approach to analyze the challenges faced by decision makers and society in general in two convergent conservation policies implementation in urban contexts in Mexico: environmental services or hydrological ecosystems services and protected natural areas. Components of a sociopolitical approach are theoretically and methodologically delineated to analyze both conservation policies in recently published academic literature. It is studied both public policy instruments and social processes and interactions they bring about with their implementation to achieve socioecosystems conservation in urban contexts in Mexico. It is concluded that it is essential to study these topics in depth.
\end{abstract}

Palabras clave: aproximación sociopolítica, políticas de conservación, servicios ambientales hidrológicos, servicios ecosistémicos, áreas naturales protegidas urbanas, México.

Keywords: Sociopolitical approach, conservation policies, hydrological environmental services, ecosystem services, urban natural protected areas, Mexico.

* Doctora en Ciencias Políticas y Sociales con Orientación en Sociología por la Universidad Nacional Autónoma de México. Investigadora de tiempo completo, Centro Regional de Investigaciones Multidisciplinarias, Área de Estudios Socio-Ambientales | gdlm@correo.crim.unam.mx 


\section{Introducción}

E

n el contexto actual de creciente degradación ambiental que experimentan los ecosistemas a nivel global, la conservación y mantenimiento de los entornos naturales ocupa un lugar relevante en los procesos de toma de decisiones $\mathrm{y}$ en el manejo del territorio, especialmente en entornos urbanos, debido a que casi la mitad de la población mundial habita en ciudades (Nelson, 2005: p. 74). México no es ajeno a esa dinámica demográfica, pues su población es eminentemente urbana. Según cifras del Instituto Nacional de Estadística, Geografía e Informatica (INEGI), para 2010 casi 78\% de la población vivía en localidades urbanas. Por ello, la decisión de conservar espacios naturales relevantes para la producción y provisión de servicios ecosistémicos resulta clave; sin embargo, las autoridades y la sociedad en general enfrentan retos para mantener las áreas naturales protegidas (ANP) con decreto, localizadas cerca o dentro de espacios urbanos, así como la provisión de diversos servicios ecosistémicos y muy en particular los hidrológicos. Esa decisión es resultado de un proceso político de toma de decisiones, que en sociedades democráticas supone la participación de una pluralidad de actores sociales además de los gubernamentales, los cuales, en teoría, a través de consensos, definen por qué es relevante conservar esos espacios, bajo qué condiciones y con qué instrumentos de política pública.

En México la conservación de la naturaleza es un asunto de interés público, por ello el gobierno federal ha implementado políticas públicas de conservación de carácter regulatorio y económico, como las declaratorias de ANP y los programas de pago por servicios ambientales, respectivamente. El interés de este trabajo es identificar y analizar, a partir de estudios académicos relevantes que relacionan los servicios ambientales o ecosistémicos hidrológicos y las ANP localizadas en espacios urbanos en México, los principales retos que enfrentan los gestores de esas áreas y otros actores sociales interesados en el tema, para lograr la conservación de los ecosistemas. El énfasis en los servicios hidrológicos relacionados con los ecosistemas forestales en contextos urbanos y su periferia se debe a que su escasez y degradación pone en peligro la viabilidad de la vida en espacios densamente poblados (Mekonnen \& Hoekstra, 2016), lo que afecta la calidad de vida de los habitantes en las ciudades. Se tiene evidencia empírica de que la urbanización ejerce una creciente presión sobre los servicios de regulación y provisión, al grado de provocar crisis sociales y políticas por la escasez del agua — tal como ocurrió en Ciudad del Cabo, Sudáfrica, con el "día cero"-, afectando a poblaciones humanas, de fauna y flora de la región (Nordling, 2018; Galicia, 2018). Para explorar la convergencia de ambas políticas de conservación 
en espacios urbanos en México en estudios científicos publicados recientemente se emplea aquí un enfoque sociopolítico. Por este medio se ha sistematizado la información más allá de tomar en consideración los instrumentos de política pública de conservación implementados, buscando identificar las acciones que demandan los actores sociales para lograr la conservación de esos socioecosistemas en contextos urbanos.

Este tema despierta interés porque los fenómenos de urbanización y metropolización están dominando los procesos demográficos y territoriales en el mundo y en México, lo que impone retos a los tomadores de decisiones y a las sociedades, de cara a la necesidad de lograr un desarrollo sostenible. En 2015, $54 \%$ de la población mundial vivía en zonas urbanas que generaban más del 80\% del piв mundial (Banco Mundial, 2015). En México 76\% de la población vive en ciudades (INEGI, 2014), esto es, en espacios complejos y dinámicos con alta concentración de infraestructura social y económica, lo que los convierte en importantes polos de desarrollo, pero también de marginación, pobreza, exclusión y contaminación.

Los entornos urbanos alteran los ciclos biogeoquímicos globales, fomentan cambios en la diversidad biológica originados en la fragmentación del hábitat y los cambios en los usos del suelo como consecuencia de la expansión urbana. El impacto socioambiental por el crecimiento de las zonas urbanas es una poderosa razón para estudiarlas, sobre todo porque una alta concentración de individuos vive en ellas y su calidad de vida depende de la adecuada gestión del territorio (Grimm et al., 2008: p. 124). En las últimas dos décadas se empezó a prestar atención al estudio de la conservación de los servicios ecosistémicos en espacios naturales de mayores dimensiones tales como las ANP en zonas urbanas a nivel internacional (Marshall et al., 2018: p. 111; Elmqvist et al., 2013; Andersson et al., 2014; Livesley et al., 2016). Sin embargo, en México estos temas se han estudiado de manera independiente, predominando más el análisis de las ANP en espacios rurales, mientras que los análisis de servicios ecosistémicos se ocupan del funcionamiento de programas y de cómo estos se perciben socialmente en espacios urbanos, lo que se discute más adelante.

Este artículo se divide en cuatro partes: en la primera se esbozan brevemente las categorías de análisis que comprende el enfoque sociopolítico y los aspectos metodológicos generales para llevar a cabo el análisis desde esa perspectiva. En la segunda, se analiza el contexto en el cual surgen las políticas de conservación de ANP y de servicios ambientales en el mundo y en México. En la tercera, se abordan las definiciones de los conceptos de servicios ecosistémicos y ambientales, los acuerdos y las polémicas generados en torno al uso de esos términos. En la última sección, y a partir de la revisión de literatura científica en la que convergen el tema de los servicios ambientales o ecosis- 
témicos y las políticas de conservación de áreas naturales protegidas urbanas en México, se discuten los retos que enfrentan los gestores de la conservación y la sociedad en general en cuanto a la implementación de ambas políticas en contextos urbanos.

\section{Enfoque sociopolítico y políticas de conservación}

El análisis político de los servicios ambientales hidrológicos en ANP puede ser entendido como el estudio de un comportamiento propositivo, intencional y planeado, que reconoce la influencia de las sociedades en la conservación y/o degradación de los ecosistemas, al tiempo que plantea estrategias y soluciones para evitar o reducir impactos. La política en general tiene un carácter relacional y es de naturaleza pública, al igual que los temas ambientales y de conservación de los ecosistemas. La política es un curso de acción con sentido, que tiene el propósito de lograr objetivos específicos a través de determinados medios (Aguilar, 2007: p. 24). Es resultado de interacciones repetidas entre actores gubernamentales y no gubernamentales interesados en el tema de la conservación, de las cuales derivan decisiones y acciones que son formuladas y reformuladas para lograr los objetivos previamente definidos.

La acción colectiva que deriva de esas interacciones es un componente clave en el análisis sociopolítico (Aguilar, 2007: pp. 24-25). A través de este enfoque, se busca visibilizar la perspectiva social o relacional que deriva en acciones que llevan a cabo distintos actores sociales más allá de los gubernamentales. Es decir, que además de reconocer las técnicas e instrumentos de política implementados a través de las políticas de gobierno (leyes, políticas públicas sectoriales, programas, etc.) para lograr la conservación de los socioecosistemas, se contempla la participación de otros actores: individuos, grupos y organizaciones. De este modo se reconoce que la toma de decisiones acerca de estos temas, no es estática, ya que ocurre en contextos societales complejos en los que participan actores sociales y políticos con sus propios puntos de vista sobre el mismo tema (Aguilar, 2007: p. 115; Lascoumes \& Le Gales, 2007: p. 2; Mann \& Simons, 2015) y quienes no necesariamente logran consensos de manera rápida y contundente para concretar la conservación de los espacios naturales que proveen los servicios ambientales o ecosistémicos en contextos urbanos.

Las políticas de conservación de la naturaleza son resultado de procesos sociopolíticos complejos que se construyen y transforman a lo largo del tiempo y a distintas escalas, en los que participan diferentes actores sociales tanto en su formulación y aplicación, como en su refutación y transforma- 
ción. El enfoque sociopolítico enfatiza en la expresión de los procesos sociales que ocurren a partir de la puesta en marcha de los instrumentos de política pública (Carsten \& Simons, 2015; Van den Broeck, 2008; Lascoumes \& Le Gales, 2007). Lascoumes \& Le Gales (2007: p. 2) señalan que los instrumentos de política se han analizado como elementos periféricos en la comprensión de las políticas públicas y en contextos sociales crecientemente complejos, se generan acumulativamente programas y políticas de diferentes sectores en los que interviene el Estado, así como otros actores no gubernamentales, lo que produce dinámicas de interacción social que van más allá del establecimiento de regulaciones, aplicación de impuestos, celebración de contratos y comunicaciones, etc. Esos instrumentos se relacionan con estilos de hacer política y formas de interacción entre actores; que no son excluyentes entre sí, puesto que pueden estar presentes diversos instrumentos y formas de interacción en un mismo espacio y tiempo, produciendo resultados que no necesariamente serán cooperativos o convergentes. En el cuadro 1 se describen brevemente las categorías que se analizan en este trabajo.

\section{Cuadro 1. Categorías analíticas para una aproximación sociopolítica}

\begin{tabular}{lll}
\hline Estilo de política & Tipo de instrumento & Tipo de interacción entre sociedad y Estado \\
\hline $\begin{array}{l}\text { Jerárquica y } \\
\text { centralizada }\end{array}$ & $\begin{array}{l}\text { Regulatorio (leyes, } \\
\text { decretos, normas, } \\
\text { programas, impues- } \\
\text { tos, etc.) }\end{array}$ & $\begin{array}{l}\text { El Estado es el actor principal en la toma de decisiones para regular lo } \\
\text { que está permitido y lo que no, induciendo así a comportamientos es- } \\
\text { pecíficos en los ciudadanos. Asimismo, es el encargado de establecer } \\
\text { las medidas de redistribución de los ingresos obtenidos }\end{array}$ \\
$\begin{array}{lll}\text { Descentralizada, } \\
\text { competitiva y } \\
\text { autónoma }\end{array}$ & $\begin{array}{l}\text { De mercado (de ca- } \\
\text { rácter contractual) }\end{array}$ & $\begin{array}{l}\text { privados-privados. Sus interacciones se centran en la eficiencia y la } \\
\text { competencia, y establecen acuerdos contractuales para ejecutar inter- } \\
\text { cambios de bienes y servicios. El Estado no tiene un papel preponde- } \\
\text { rante en los procesos de toma de decisiones. }\end{array}$ \\
$\begin{array}{lll}\text { Democrática y } \\
\text { participativa }\end{array}$ & $\begin{array}{l}\text { Acuerdos de jure o o } \\
\text { de facto }\end{array}$ & $\begin{array}{l}\text { mentales bajo un esquema de una horizontalidad relativa. La interac- } \\
\text { ción entre actores puede generar redes de acción social y política que } \\
\text { contribuyen al intercambio de información y comunicación. El Estado } \\
\text { participa como regulador en estas interacciones }\end{array}$ \\
\hline
\end{tabular}

Fuente: Elaboración propia con información de Lascoumes \& Le Gales (2007), Carsten \& Simons (2015) y Meuleman (2010).

Los instrumentos y las acciones que se originan con la interacción entre actores sociales pueden combinarse en un mismo ámbito y pueden ser procesos inacabados y en construcción. Tal como ocurre en los aspectos que se analizan en este trabajo, ya que se contemplan dos instrumentos de conservación 1) de carácter regulatorio, es decir, los decretos de áreas naturales protegidas; $y$ 2) económico, a través de los programas de pago por servicios ambientales de la Comisión Nacional Forestal, que aunque actualmente es un programa de subsidios, el propósito de esta política es lograr la generación de mercados, tal como se ha establecido política y normativamente. Ambos instrumentos de política 
han orientado un determinado tipo de interacciones entre actores sociales, las cuales están reguladas por marcos institucionales o normativos; pero también existen elementos culturales, históricos, simbólicos y sociales que inciden en la puesta en marcha y funcionamiento de esos instrumentos.

En México se ha estudiado poco la convergencia de las políticas de conservación de ANP y los programas de pago por servicios ecosistémicos hidrológicos en contextos urbanos. No obstante, la literatura científica nos ofrece estudios interesantes que son relevantes en los que convergen los temas de "ANP", "servicios ecosistémicos o ambientales hidrológicos", "espacios urbanos" en "México". Dichos estudios se obtuvieron en las bases de datos científicas: Scopus y Redalyc. La búsqueda se realizó considerando que esos términos estuvieran en el título, en las palabras claves y en el resumen. El material encontrado se revisó de manera cuidadosa, identificando los retos señalados por los autores para lograr la conservación en espacios urbanos y el tipo de interacciones a que inducen los instrumentos de política pública de conservación analizados.

\section{Contexto de las políticas de conservación: entre ANP y servicios ambientales}

A partir de la segunda mitad del siglo $\mathrm{xx}$, el vertiginoso deterioro ambiental y sus consecuencias para la vida, salud y seguridad humanas y los ecosistemas, ha contribuido a incorporar en las agendas de política ambiental internacional, nacional y local el tema de la conservación de las ANP y su importancia para la provisión y mantenimiento de diversos servicios ambientales, especialmente los hidrológicos. Este interés también ha sido promovido e impulsado de manera contundente por la Organización de las Naciones Unidas (ONU) a través de conferencias internacionales, programas, convenios, protocolos y celebraciones que los países miembros suscriben y que se obligan a cumplir, además de incorporar los principios y compromisos a favor del cuidado del medio ambiente en sus marcos institucionales nacionales.

La protección de espacios naturales en México no es una preocupación nueva, pues se ha practicado desde la época precolombina mediante jardines botánicos, zoológicos y zonas de protección de bosques circundantes a asentamientos humanos. Sin embargo, durante el periodo colonial el uso intensivo de los recursos naturales modificó a gran escala los ecosistemas (Simonian, 1999). Fue en el siglo XIX que surgió en Europa una corriente de pensamiento preocupada por la destrucción de los bosques que permeó en América Latina. La política de áreas naturales protegidas en México inició oficialmente en 1898 con el de- 
creto del Bosque Nacional Monte Vedado del Mineral El Chico, en Hidalgo, ${ }^{1}$ publicado en el Diario Oficial el 22 de junio de ese mismo año (Conanp, 2005: p. 9). La finalidad de esta declaratoria era conservar los recursos forestales y lo que hoy se conoce como servicios ecosistémicos.

A lo largo del siglo xx se impulsaron acciones de conservación, restauración y reforestación en zonas forestales, así como el establecimiento de reservas forestales y parques nacionales para proteger cuencas hidrológicas, bosques y selvas, paisajes, áreas naturales para la recreación, etcétera (De la Maza et al., 2003: pp. 17-18; Simonian, 1999). En especial durante la presidencia de Lázaro Cárdenas (1934-1940) se decretaron por todo el país áreas naturales protegidas en ecosistemas forestales relevantes para la provisión de servicios ecosistémicos. Tales declaratorias no siempre fueron respetadas ni por los pobladores, ni por las autoridades. Además, al determinarlas de manera aislada, se favoreció el manejo fragmentado de los ecosistemas y los territorios.

Con las declaratorias, el gobierno emite regulaciones que restringen y regulan los usos del territorio y sus recursos naturales, lo que ha causado conflictos sociales y políticos, ya que omiten las opiniones y derechos de la población local sobre el territorio. Esto se ha documentado ampliamente sobre todo en las zonas rurales e indígenas (De la Maza et al., 2003; West et al., 2006; Paré \& Fuentes, 2007; Paré \& Robles, 2008; Bezaury-Creel \& Gutiérrez, 2009: p. 391; Andrade, 2009: p. 52). En contraste, en espacios urbanos la sociedad civil y los habitantes locales (vecinos, en muchos casos) han sido los principales promotores de las declaratorias de protección, argumentando que la falta de espacios verdes o naturales afecta su calidad de vida. A nivel mundial, los tomadores de decisiones y las autoridades gubernamentales se han ocupado crecientemente de integrar las actividades de las comunidades con la conservación de áreas naturales protegidas, con el fin de fomentar actitudes proambientales y coparticipativas (Dos Santos \& De la Corte Bacci, 2017; Ribeiro \& Ribeiro, 2016; Ernstson et al., 2008). En paralelo, actores de la sociedad civil cuestionan a las autoridades por permitir el uso de áreas no urbanizadas y relevantes para el mantenimiento y provisión de servicios ambientales o ecosistémicos, como espacios disponibles para el crecimiento urbano, lo que genera conflictos socioambientales ${ }^{2}$ en

1 El decreto buscaba conservar los servicios recreativos que El Chico prestaba a la población local y de otras regiones, además de que contribuía a la captación pluvial y a la recarga de acuíferos para Pachuca, la capital del estado de Hidalgo (Conanp, 2005: p. 12).

2 En su análisis sobre conflictos socioambientales, Paz (2014: p. 42) contabiliza 71 casos urbanos en México, los cuales están vinculados a la urbanización-metropolización, especulación inmobiliaria, privatización de servicios públicos, construcción de centros comerciales y recreativos en espacios públicos o en zona de fragilidad ambiental, construcción de desarrollos inmobiliarios en bosques, cerros, humedales y tierras de cultivo que rodean las ciudades y corrupción pública y privada, entre otros. 
espacios disputados social y políticamente. Las consecuencias se traducen en el crecimiento de las ciudades sin planeación, en zonas de fragilidad ambiental, poniendo en riesgo la seguridad y la salud de los habitantes locales, así como la permanencia de los ecosistemas (Paz, 2014: p. 44; De la Mora-De la Mora \& Montaño, 2016).

La Organización Mundial de la Salud (OMS) ${ }^{3}$ reconoce que es indispensable que los habitantes de las ciudades dispongan de áreas verdes y parques urbanos ya que estos fomentan el encuentro social, embellecen y dan plusvalía al entorno, permiten la retención de agua, ayudan a disminuir los contaminantes atmosféricos y a la regulación climática, además de reducir el estrés y la depresión, entre otros temas de relacionados con la salud humana (Díaz, 2012). De igual modo, la pérdida o ausencia de esos espacios refleja la crisis ambiental y social que experimentan los habitantes de las ciudades (Flores-Xolocotzi \& González-Guillén, 2010).

En los últimos 25 años la declaratoria de ANP se ha transformado en la columna vertebral de la estrategia mexicana para la conservación de los ecosistemas y su biodiversidad (De la Maza, 2010: p. 18). Pero la falta de recursos humanos y financieros ha dificultado su mantenimiento (Simonian, 1999: p. 227; Pagiola et al., 2004: p. 6; Bezaury-Creel \& Hernández, 2010: p. 398). Ante las limitaciones de dicha política, la alternativa ha sido la implementación de instrumentos económicos o de mercado para la conservación de los ecosistemas, y los servicios ambientales o ecosistémicos (MEA, 2005b; Wunder, 2005; Wunder et al., 2005).

En México, la política de ANP se complementa desde 2003 con los Programas de Pago por Servicios Ambientales (hidrológicos y de biodiversidad). La finalidad de estos es valorar los bienes y servicios de los ecosistemas forestales para ofrecer opciones de desarrollo y mejorar la calidad de vida de los dueńos y poseedores de los recursos forestales, al tiempo que se busca la conservación de los ecosistemas evitando la deforestación (Torres, 2010: p. 102).

La declaratoria de ANP y las estrategias de conservación de los servicios ambientales y ecosistémicos persiguen el mismo propósito pero por diferentes medios: la conservación de los ecosistemas. Normativamente, la Ley General del Equilibrio Ecológico y Protección al Ambiente (LGEEPA) determina los objetivos de las ANP: la preservación de ambientes naturales representativos de regiones biogeográficas y ecológicas, y ecosistemas frágiles; la salvaguarda de la diversidad genética de especies silvestres; el aprovechamiento sustentable de los

3 La oms recomienda una superficie mínima de áreas verdes por habitante en las ciudades de $9 \mathrm{~m}^{2}$, y accesibles a quince minutos caminando desde las viviendas. Esto no se cumple en las grandes ciudades mexicanas (Flores-Xolocotzi \& González-Guillén, 2010). 
ecosistemas; y la protección de zonas forestales en montañas donde se originan torrentes y el ciclo hidrológico de las cuencas, entre otros (LGEEPA, arts. 4445bis). Por su parte, los servicios ambientales se refieren a los beneficios tangibles e intangibles producidos por los ecosistemas, que son necesarios para la supervivencia del sistema natural y biológico en su conjunto y del propio ser humano (LGEEPA, art. 3).

La Secretaría de Medio Ambiente y Recursos Naturales (Semarnat) como cabeza del sector ambiental en México y la Comisión Nacional Forestal (Conafor), son las instancias gubernamentales encargadas de promover el aprovechamiento sustentable de los recursos naturales y la conservación de los servicios ambientales de los ecosistemas forestales, respectivamente. La Conafor, a través del programa Pago por Servicios Ambientales Hidrológico, promueve que las poblaciones urbanas, entre otras, valoren los ecosistemas forestales para mantener su calidad de vida y revertir los procesos de deterioro debidos a la actividad humana en esas zonas (Conafor, 2016).

La estrategia planteada por la Conafor se sustenta normativamente en la Ley General de Desarrollo Forestal Sustentable (LGDFs) — su más reciente reforma se publicó en el Diario Oficial de la Federación el 5 de junio de 2018-, para "coadyuvar en la definición y promoción de mercados de bienes y servicios ambientales" (LGDFs, art. 20, fracc. X). Esta ley especifica que la federación tiene la atribución de promover y proponer "la incorporación de los costos relacionados con la conservación de los recursos forestales para el beneficio de la sociedad en las actividades productivas para establecer medios de compensación y conservación de los servicios ambientales derivados de los ecosistemas forestales" (LGDFS, art. 10, fracc. XII); y "diseñar y establecer, dentro de las Entidades de la Administración Pública Federal, mecanismos para incorporar los costos relacionados con la conservación de los recursos forestales para el beneficio de la sociedad en la instrumentación de medios de compensación de los bienes y servicios ambientales" (LGDFs, art. 10, fracc. XIII). Adicionalmente se establece como criterio obligatorio de la política forestal "la valoración de los bienes y servicios ambientales" y "el apoyo, estímulo y compensación de los efectos económicos de largo plazo de formación del recurso forestal y del costo de bienes y servicios ambientales" (art. 33, fracc. XIII y XIV, respectivamente). Las críticas a este planteamiento han sido que la valorización económica de la conservación de los ecosistemas no resuelve la degradación de estos, ni desincentiva la deforestación de manera automática tal y como se analizará en la última parte de este trabajo. En consecuencia, la generación de mercados parece que no funciona para asegurar el mantenimiento de los bosques y selvas en el largo plazo. Esta polémica también ha estado presente en el desarrollo conceptual de los servicios ambientales o ecosistémicos. 


\section{Servicios ambientales y ecosistémicos: debates principales}

Los conceptos servicios ecosistémicos y servicios ambientales se han socializado fuertemente en la última década; sin embargo, su definición sigue siendo abstracta y poco operativa (De Sartre et al., 2014: p. 13; Wallace, 2007) ${ }^{4}$ y en ocasiones ambigua, lo que repercute en la producción de conocimiento, en la movilización de actores en torno al tema, y en la generación de acciones y políticas públicas ambientales internacionales y locales, que favorecen un determinado tipo de políticas y procesos de toma de decisiones que llegan a ser difusos. Es el caso de la política pública de la Conafor, que normativamente plantea crear mercados de servicios ambientales, pero en los hechos es un subsidio gubernamental.

Los términos servicios ecosistémicos y servicios ambientales se utilizan indistintamente pero no significan lo mismo. Los primeros se refieren al conjunto de organismos y condiciones abióticas y sus interacciones que producen beneficios para los seres humanos. Se trata de una definición que contempla múltiples componentes de los ecosistemas que no pueden ser desagregados (Balvanera $\&$ Cotler, 2007: p. 10; Wunder, 2005: p. 4). Mientras que los segundos se refieren a la naturaleza separable de los servicios sin prestar atención a las interacciones necesarias para su provisión y conservación. Este último concepto es más utilizado por los tomadores de decisiones (Wunder, 2005: p. 4; Balvanera \& Cotler, 2007: p. 10). Aunque independientemente del concepto utilizado, los estudios tienden a analizar por separado los distintos servicios.

Por otra parte, el discurso que permea el uso de ambos conceptos valida el vínculo entre ecosistemas y bienestar humano, aunque se reconoce que aún es insuficiente el conocimiento científico para determinar cómo y en qué medida inciden uno en el otro (Balvanera \& Cotler, 2007: 118; Chan et al., 2006). Según De Sartre et al. (2014: p. 15), el término servicios ecosistémicos (y agregamos el de servicios ambientales) remite a una noción de frontera, ya que ambos conceptos se construyen y cobran sentido a partir de la retroalimentación entre ciencia, acción social y quehacer político. Estos conceptos pueden ser puente o interfaz entre disciplinas científicas como la ecología, la sociología, la ciencia política y la economía, entre otras; las cuales conjuntamente buscan solucionar problemas socioambientales complejos mediante la innovación científicotécnica y la generación de metodologías, teorías y nuevos conceptos aplicables a realidades socioambientales específicas.

Entre las críticas a dichos conceptos se encuentran el antropocentrismo y la unidireccionalidad del enfoque, pues privilegian la observación de las funciones

4 Wallace (2007) señala una ambigüedad en el uso de términos clave como procesos, funciones y servicios ecosistémicos, por lo que desarrolla una clasificación después de discutir estos conceptos. 
y procesos de los ecosistemas como bienes y servicios en beneficio de la sociedad (De Groot et al., 2002; Wallace, 2007), mas no consideran las relaciones recíprocas entre las comunidades humanas y ecosistemas (Norgaard, 2013: p. 2; Comberti et al., 2015). También se ha señalado la tendencia a concebir esas funciones y procesos desde una óptica utilitarista enfocada en la valoración económica y de mercado (Gómez-Baggethun et al., 2010; Dempsey \& Robertson, 2012; Rival \& Muradian, 2013: p. 1; Fischer \& Brown, 2014).

Entre los aciertos reconocidos en el uso de estos conceptos se halla el que colocan en el centro de la discusión política la relación interdependiente entre naturaleza y sociedad, incorporando estos términos, al menos discursivamente, en las agendas de política ambiental globales y domésticas, apelando a la necesidad de producir conocimiento desde una perspectiva multi, trans e interdisciplinaria (Schröter et al., 2014; Tress et al., 2004).

Por otro lado, los temas de conservación, ya sea que se relacionen con el establecimiento de ANP o con los servicios ambientales o ecosistémicos, han sido incorporados en instrumentos internacionales de carácter vinculante, lo que contribuye al avance e implementación de políticas de conservación a nivel doméstico en países desarrollados y en desarrollo. La conservación cobra particular importancia a partir de la Conferencia de las Naciones Unidas sobre el Medio Humano de Estocolmo en 1972. Diez años después, a raíz del aniversario de Estocolmo, el Consejo de Gobierno del Programa de Medio Ambiente de las Naciones Unidas solicitó una revisión de los avances alcanzados hasta entonces (Holdgate et al., 1982). Así, en 1992 la Conferencia de Río de Janeiro contribuyó a fortalecer esos temas en las agendas políticas internacionales y nacionales. Y desde 2005, con la iniciativa de la Evaluación de los Ecosistemas del Milenio de las Naciones Unidas, se consolida el uso del concepto de servicios ecosistémicos y ambientales en sectores académicos y de políticas públicas ambientales, lo que se evidencia en la creciente producción de conocimiento científico al respecto, y en la implementación de políticas públicas de conservación de los ecosistemas forestales y los servicios ecosistémicos asociados (Balvanera \& Cotler, 2007: p. 10; Castro \& De Sartre, 2014: p. 50; Perevochtchikova \& Oggioni, 2014). Ambas políticas de conservación convergen en la práctica; sin embargo, se deben destacar los retos que proponen los estudios que relacionan estos temas en el ámbito urbano.

\section{Conservación de ANP y servicios ecosistémicos en zonas urbanas en México}

Los estudios que analizan los casos de áreas naturales protegidas urbanas relevantes para la conservación de los servicios ambientales hidrológicos se refie- 
ren fundamentalmente a los de la Ciudad de México, Xalapa en Veracruz, la ciudad de Puebla, Huixquilucan en el Estado de México y a Guadalajara en Jalisco. Allí se abordan la (in)definición e (in)cumplimiento de las políticas ambientales y urbanas; las consecuencias de esas omisiones en el ambiente (contaminación de suelo, agua y atmósfera); la implementación de mecanismos de compensación por servicios ambientales en ANP periurbanas y sus efectos sociales, y la identificación de los servicios ecosistémicos hidrológicos en calidad y cantidad, entre otros.

Los análisis de Schteingart (2006), Aguilar \& Santos (2011), Marín et al. (2013) y García (2014) muestran que la decisión de declarar áreas de protección y conservación en zonas urbanas o periféricas a las ciudades tiene como propósito mantener y preservar la provisión de servicios ambientales vitales para la población local, pero, sobre todo, imponer límites al crecimiento urbano. Tales declaratorias no siempre cumplen el objetivo de conservar los ecosistemas pues las políticas gubernamentales tienden a ser reactivas, inconsistentes y permisivas; además de ignorar la necesidad de aplicar otras políticas, como la de vivienda, para atender las demandas sociales de modo planeado.

Las inconsistencias jurídicas, la ineficiencia administrativa, la descoordinación en el diseño y aplicación de la legislación urbana y ambiental, más la corrupción de actores públicos y privados, han propiciado la expansión urbana en zonas de alto valor ecológico (Schteingart, 2006; Aguilar \& Santos, 2011). Fernández et al. (2002), Aguilar \& Santos (2011), Schteingart (2006) y MerlínUribe et al. (2013) han documentado y analizado los cambios de uso de suelo al sur de la Ciudad de México en zonas de conservación; mientras que Benítez et al. (2012) y Hernández \& Torres (2015) documentan el mismo tema en Xalapa, Veracruz. Todos estos autores registran la creciente degradación en zonas periféricas de relevancia ecológica a causa de los asentamientos irregulares. Para ello, utilizan información espacial y sistemas de información geográfica con el propósito de mostrar la urbanización descontrolada y sin planeación en las periferias urbanas.

Otros autores se ocupan de los servicios ecosistémicos potenciales, reales y percibidos, que aún brindan los ecosistemas naturales en las ciudades pese a su degradación. En particular, Silke et al. (2008) analizan los servicios ambientales potenciales del suelo de conservación de la Ciudad de México, es decir: recarga de acuíferos e infiltración de agua, lo que reduce la escorrentía, evita inundaciones y arrastre de basura hacia las partes bajas, y ayuda al almacenamiento de agua y a aliviar la sobrecarga del drenaje artificial. Asimismo, estos autores proponen la protección de fragmentos de suelo libre de construcciones adjudicándoles la función de área verde, a fin de incrementar el potencial de captura de carbono, producción de oxígeno, el control de humedad y tempera- 
tura, e incluso el incremento del área urbana de recreación. Aunque los autores no abordan explícitamente las áreas naturales protegidas urbanas, en su análisis subyace el reconocimiento del potencial del suelo para mantener los ecosistemas aun en espacios urbanizados.

Almeida-Leñero et al. (2007) describen los servicios ecosistémicos que provee la cuenca del río Magdalena en la Ciudad de México, con el propósito de lograr su protección y adecuado manejo. Por su parte, Jujnovsky et al. (2010), Mazari-Hiriart et al. (2014) y Chávez (2015) estudian la cuenca del río Magdalena-Eslava (último río superficial que provee de agua a la ciudad). En los primeros dos estudios, los autores analizan la cantidad y calidad del agua, mostrando el grado de contaminación del recurso y recomiendan rehabilitar el río y replicar esas acciones en otros lugares por razones de salud pública. En el tercer estudio, la autora identifica la valoración del entorno natural por parte de los ejidatarios quienes viven en la cuenca del río Eslava y encuentra que los servicios ambientales más reconocidos por ellos son la provisión de alimento y agua, el aire limpio, los escenarios placenteros y la regulación del clima. En contraste, los servicios ecosistémicos menos significativos parecen ser el filtrado de partículas y compuestos, el mantenimiento de la diversidad biológica y la información cultural y artística.

Por otro lado, Nava-López et al. (2016) analizan la calidad del agua en la presa Guadalupe en la Ciudad de México y reconocen que es necesario estudiar el manejo de los servicios ecosistémicos riparios en megaciudades, a fin de entender las conexiones entre la calidad del agua superficial y factores del paisaje como los usos del suelo y la topografía. Los autores muestran que la elevación del terreno influye en la calidad del agua, ya que la topografía determina el tipo de actividad humana. Esa información es valiosa para los tomadores de decisiones, ya que da la pauta para elegir acciones y políticas de manejo territorial en contextos con vacíos regulatorios en megaciudades de países como México.

Los estudios que abordan la franca interacción de las áreas naturales protegidas con las zonas urbanas coinciden en que los principales problemas son la progresiva invasión humana, pues se utilizan como tiraderos de basura y deshechos o como zonas de expansión urbana y habitacional por parte de vecinos y autoridades. Asimismo, se evidencia el abandono y descuido de las autoridades encargadas de su administración y gestión, sobre todo en ANP bajo administración de gobiernos locales (Almeida-Leñero et al., 2007; Tlapa, 2011; Hernández \& Torres, 2015; De la Mora-De la Mora, 2015). Lo anterior muestra que incluso las políticas jerárquicas que centralizan los procesos de toma de decisiones no han logrado sus objetivos, ya que es notoria la falta de control por parte del Estado para regular los usos y el acceso a dichos espacios. Inclusive el establecimiento de las áreas naturales protegidas urbanas a veces proviene de 
una demanda de la sociedad civil para mantener esos espacios, ante la falta de compromiso e interés de los encargados gubernamentales (De la Mora-De la Mora \& Montaño 2016; García, 2014). De igual forma, es común que no haya información precisa sobre los límites de las ANP, lo que impide una evaluación adecuada de sus dinámicas y el ordenamiento territorial, lo que repercute en el cabal cumplimiento de la normatividad ambiental para regular el uso y manejo de los recursos naturales, a lo que se suma la falta de coordinación entre autoridades y propietarios (Tlapa, 2011; Hernández $\&$ Torres, 2015).

Los problemas que se identifican en la literatura acerca de las ANP se resumen en Hernández \& Torres (2015), autores que apuntan que en las ANp Cerro de la Galaxia y Molino de San Roque, en la zona metropolitana de Xalapa, se observa la recuperación de vegetación o cobertura arbórea en zonas de difícil acceso, mientras que el deterioro comienza en las colindancias con al área urbana. Estos autores reconocen que la regeneración en algunas zonas es por sucesión secundaria y no por un manejo planificado, y concluyen que, si esos espacios no estuvieran protegidos, ya hubieran sido devorados por la urbanización.

El manejo de las áreas periurbanas es complejo, entre otros aspectos, por la convergencia de los ámbitos rural y urbano, cuyas políticas sectoriales no siempre son compatibles (Pérez-Campuzano et al., 2016). En investigaciones sobre la implementación de políticas y programas de conservación forestal en comunidades propietarias de bosques localizados en el suelo de conservación de la Ciudad de México, que específicamente se refieren al Programa de Pago por Servicios Ambientales Hidrológico de la Conafor, se encontró que los beneficiarios perciben que mientras prosigan los subsidios o se tenga acceso a esos recursos económicos, los bosques se seguirán conservando; pero si esto cambia, su decisión no podrá mantenerse (Pérez-Campuzano et al., 2016; Caro-Borrero et al., 2015; Balderas et al., 2013). Asimismo, los beneficiarios del programa consideran que el dinero que reciben es insuficiente para desalentar el cambio de uso de suelo y evitar la deforestación. En otras palabras, el programa no promueve la existencia de una iniciativa real de participación voluntaria para invertir en y para el bosque (Caro-Borrero et al., 2015a; Perevochtchikova $\&$ Vázquez, 2012).

Balderas et al. (2013), por su parte, consideran que los mecanismos de pago por servicios ambientales necesitan ser atractivos para los propietarios de los recursos forestales, sobre todo en ANP periurbanas, para que se asegure su participación. Para demostrarlo, llevaron a cabo un estudio con propietarios privados y ejidatarios en el Anp Bosque La Primavera en Guadalajara, Jalisco; donde muestran que la dependencia e importancia que dan los propietarios al dinero de los pagos por servicios ambientales decrece si se promueven acciones de desarrollo local para mejorar servicios de salud y educación, además de incluir la 
generación de empleos y proyectos productivos. Asimismo, demuestran que la disponibilidad a participar en el programa crece en la medida que aumenta la distancia de la zona urbana de Guadalajara. Por lo anterior, recomiendan ofrecer una combinación de beneficios monetarios y no monetarios basados en las necesidades de desarrollo local, para promover la participación en el programa PSA de la Conafor o de cualquier otro mecanismo de compensación económica que desaliente la deforestación.

Por otro lado, desde la perspectiva de la planeación urbana se ha comenzado a combinar la construcción de infraestructura con la inclusión de espacios verdes y naturales, ya que social y ambientalmente los espacios públicos representan un gran valor para el esparcimiento, la salud de las personas y la provisión de servicios ambientales (Flores-Xolocotzi \& González-Guillén, 2010). Los espacios verdes suelen tener una distribución heterogénea en las ciudades, lo que puede traducirse en segregación social debido a la desigual distribución de la cobertura vegetal y su relación con el nivel de ingresos de los habitantes. Por ejemplo, Romero et al. (2012: pp. 85-86) señalan que la vulnerabilidad física se vincula con la vulnerabilidad social, la cual depende de la situación socioeconómica de la población urbana. En Guadalajara, el oriente concentra cerca del $4 \%$ de la vegetación urbana y la parte occidental el $22 \%$, lo que puede relacionarse con la mayor o menor concentración de ozono, y la reducción de emisiones de $\mathrm{CO}^{2}$ y las altas temperaturas (Pimienta-Barrios et al., 2014), aspectos relevantes para hacer notar a la sociedad y a los tomadores de decisiones sobre la necesidad de fomentar y mantener esos espacios por los beneficios que aporta a la salud pública, física y emocional de los ciudadanos.

En la literatura revisada se demuestra que el crecimiento urbano caótico desafía constantemente los límites de las ciudades, invadiendo y degradando las áreas naturales protegidas decretadas o los espacios naturales existentes en las ciudades o en su periferia. Sin embargo, la sociedad civil participa y enfrenta este problema directamente frente a las autoridades, proponiendo, impulsando y defendiendo la declaratoria de nuevas ANP. Comunidades de vecinos, académicos y activistas se organizan para lograr que estos espacios mantengan su vocación pública y ambiental frente a la vorágine del crecimiento inmobiliario que favorece a grupos de actores privados interesados en generar espacios de consumo (De la Mora-De la Mora \& Montaño, 2016; García, 2014; Benítez et al., 2012). La sociedad civil exige la puesta en marcha de esquemas participativos que favorezcan procesos de toma de decisiones más democráticos, para ello generan redes de interacción e intercambio de información entre actores no gubernamentales interesados en mantener esos espacios.

Sectores de la sociedad civil prestan atención a la conservación; sin embargo, los vacíos normativos y la toma de decisiones errática de quienes se encargan de 
diseñar e implementar las políticas públicas, someten la permanencia de estos espacios a las fuerzas del mercado más que al interés social y ambiental, lo que produce conflictos y disputas por mantener áreas que indiscutiblemente son vitales para las urbes y sus ciudadanos. La desatención de estos espacios y los servicios ambientales que proveen, tanto por las autoridades como por la sociedad en general, incrementa las posibilidades de escenarios de crisis socioambientales a causa de la escasez de recursos vitales como el agua o por el incremento de los riesgos causados por la degradación de las ANP que tienen un valor ecológico y social vital, pero que cuesta reconocer políticamente.

\section{Conclusión}

La conservación y el buen funcionamiento de los ecosistemas naturales urbanos constituye la base de un desarrollo territorial equilibrado, puesto que influyen positivamente en el bienestar humano y son indispensables para llevar a cabo cualquier actividad social. Las ANP urbanas y periurbanas, como garantes de valores públicos y sociales, deben gozar de mayor visibilidad política en la toma de decisiones en materia territorial, urbana y ambiental, para asegurar su permanencia. En este contexto, la instrumentación convergente de políticas de conservación de servicios ambientales o ecosistémicos en ANP puede contribuir a revalorar social y políticamente su importancia estratégica, lo que tendría resonancia en la planeación urbana, así como en otras políticas públicas relacionadas por ejemplo con el tema de la vivienda, el sector agropecuario, hidráulico y forestal de las zonas periurbanas.

Aunque existen sectores sociales que reconocen que estos espacios sustentan el desarrollo local, políticamente no se ha dado prioridad a la creación de instrumentos de política pública (normas, programas o estrategias), que atiendan integradamente las causas de la degradación de los servicios ambientales en las ANP. Asimismo, la administración pública no cuenta con instancias especializadas en el diseño e implementación de acciones y políticas sostenibles, además los tomadores de decisiones ignoran este tema.

Por otro lado, los estudios analizados apuntan los vacíos y contradicciones institucionales en materia ambiental y urbana, así como el incumplimiento de normas e instrumentos de planeación y ordenamiento territorial. De igual forma, la falta de organización de los actores locales, la desconfianza hacia las autoridades y la presencia de conflictos de interés entre grupos sociales y económicos, se manifiestan en el territorio produciendo más desorden.

Aunque son evidentes los esfuerzos a través del estudio e implementación de políticas y programas que pretenden desalentar los cambios de uso de suelo y la 
deforestación en espacios naturales localizados en zonas urbanas, actores privilegiados y con poder en los procesos de toma de decisiones fomentan la especulación inmobiliaria, con lo que favorecen la urbanización en detrimento de la conservación de los ecosistemas y los servicios ambientales que proveen. En tanto que las compensaciones económicas que se otorgan a los propietarios de los recursos forestales para que los conserven, son insuficientes e ineficaces para desincentivar el cambio de uso de suelo, por lo que la amenaza de deforestación no desaparece ni tampoco mejora la calidad de vida de los propietarios de los bosques.

La conservación entendida como un curso de acción con sentido necesita integrar y reconciliar la política ambiental y urbana, pero con un enfoque público y social, que fomente la corresponsabilidad y reconozca que el bienestar y calidad de vida de todos los actores urbanos depende de la existencia y permanencia de las ANP. Estos ámbitos no pueden permanecer independientes ni ajenos entre sí, ni mucho menos responder a procesos económicos que inducen a la inequidad social y a la degradación ambiental. Por el contrario, deben ser considerados elementos interactuantes y complementarios en la formulación de programas y políticas de manejo del territorio fomentadas por el Estado, pero que promuevan y faciliten el involucramiento de la sociedad. Así, el enfoque sociopolítico de la conservación de los servicios ambientales hidrológicos y su relación con las ANP urbanas es un campo de análisis que merece estudiarse con mayor profundidad, puesto que las acciones de conservación tienen su origen en decisiones y acciones políticas que deben hacer prevalecer el interés público y social.

\section{Referencias}

Aguilar A. G. \& Santos, C. (2011, octubre). Informal settlements needs and environmental conservation in Mexico City: An unsolved challenge for land-use-policy. Land use policy. Scientific Research An Academic Publisher, 28(4), 649-662. doi: dx.doi.org/10.1016/j .landusepol.2010.11.002

Aguilar Villanueva, L. F. (2007). Estudio Introductorio. La hechura de las políticas (pp. 3-29). México: Porrúa.

Almeida-Leñero, L., Nava, M., Ramos, A., Espinosa, M., Ordoñez, M. \& Jujnovsky, J. (2007, julio-diciembre). Servicios ecosistémicos en la cuenca del Río Magdalena, Distrito Federal, México. Gaceta Ecológica. Secretaría de Medio Ambiente y Recursos Naturales, (84-85), 53-64.

Andersson, E., Barthel, S., Borgström, S., Colding, J., Elmqvist, T., Folke, C. \& Gren, A. (2014). Reconnecting cities to the biosphere: Stewardship of green infrastructure and urban ecosystem services. Ambio, (43), 445-453. 
Andrade, I. (2009, abril). ¿El fin de la Frontera? Reflexiones desde el caso colombiano para una nueva construcción social de la naturaleza protegida. Revista de Estudio Sociales, (32), 48-59.

Balderas Torres, A., MacMillan Douglas, C., Skutsch, M. \& Lovett, J. C. (2013, diciembre). Payments for ecosystem services and rural development: Landowners' preferences and potential participation in western Mexico. Ecosystem Services, 6, 72-81. doi: dx.doi.org/10.1016/j .ecoser2013.03.002

Balvanera, P. \& Cotler, H. (2007, julio-diciembre). Acercamientos al estudio de los servicios ecosistémicos. Gaceta Ecológica, (84-85), 8-15. Recuperado el 14 de diciembre de 2016, de www.redalyc.org/articulo.oa?id $=53908502$

Banco Mundial. (2015). Desarrollo urbano: panorama general. Recuperado el 1 de septiembre de 2016, de http://www.bancomundial.org/es/topic/urbandevelopment/overview

Benítez, G., Pérez-Vázquez, A., Nava-Tablada, M., Equihua, M. \& Álvarez-Palacios, J. L. (2012). Urban expansion and the environmental effects of informal settlements on the outskirts of Xalapa city, Veracruz. Environment \& Urbanization, 24(1), 149-166. doi: $10.1177 / 0956247812437520$

Bezaury-Creel, J. \& Gutiérrez Carbonell, D. (2009). Áreas naturales protegidas y desarrollo social en México. En R. Dirzo, R. González \& I. J. March (Comps.), Capital natural de México. Vol. II: Estado de conservación y tendencias de cambio (pp. 385-431). México: Conabio.

Caro-Borrero, A., Corbera, E., Neitzel Kurt, C. \& Almeida-Leñero, L. (2015a, febrero). "We are the city lungs": Payments for ecosystem services outskirts of Mexico City. Land Use Policy, (43), 138-148. doi: dx.doi.org/10.1016/j.landusepol.2014.11.008

Carsten, M. \& Simons, A. (2015). Local emergence and international developments of conservation trading systems: Innovation dynamics and related problems. Environmental Conservation, 42(04), 325-334. doi: https://doi.org/10.1017/S0376892914000381

Castro, M. \& De Sartre, X. A. (2014). De la biodiversité aux services écosystémiques. Approche quantitative de la généalogie d'un dispositif. Political ecology des services écosystémiques. En X. Arnauld de Sartre, M. Castro, S. Dufour \& Johan Oszwald (Dirs.), Political ecology des services écosystémiques (pp. 49-83). Bruselas: Peter Lang.

Chan Kai, S. R., Cameron, D., Underwood, E. \& Daily, G. (2006, octubre). Conservation planning for ecosystem services. Plos Biology. doi: dx.doi.org/10.1371/journal.pbio.0040379

Chávez Cortés, M. M. (2015, enero-abril). Valoración del entorno natural de la cuenca del río Eslava, D.F. Revista Espiral, 22(62), 171-204. 
Comberti, C., Thornton, T. F., Echeverria, V. W. \& Patterson, T. (2015). Ecosystem services or services to ecosystems? Valuing cultivation and reciprocal relationships between humans and ecosystems. Global Environmental Change, (34), 247-262. doi: http://dx.doi.org/10.1016 /j.gloenvcha.2015.07.007

Conafor. (2016). Servicios Ambientales. [Última actualización: 24 de febrero de 2015]. Recuperado el 20 de noviembre de 2016, de http://www.conafor.gob.mx/web/temas-forestales/ servicios-ambientales/

Conanp. (2005). Programa de conservación y manejo Parque Nacional El Chico, México. México: Conanp. Recuperado el 10 de octubre de 2016, de http://centro.paot.org.mx/documentos/ine /chico.pdf; http:/www.conanp.gob.mx/que_hacemos/pdf/programas_manejo/PN_Chico .pdf

De Groot, R. S., Wilson Matthew, A. \& Boumans Roelof, M. J. (2002, junio). A typology for the classification, description and valuation of ecosystem functions, godos and services. Ecological Economics, 41(3), 393-408. doi: http://dx.doi.org/10.1016/S0921-8009(02)00089-7

De la Maza, R. (2010). Áreas naturales certificadas. En J. Carabias, J. Sarukhán, J. de la Maza \& C. Galindo (Coords.), Patrimonio natural de México. Cien casos de éxito (pp. 17-19). México: Conabio/Semarnat.

De la Maza, E. J., Cadena González, R. \& Piguerón Wirz, C. (2003). Estado actual de las Áreas Naturales Protegidas de América Latina y el Caribe (Versión Preliminar). Programa de las Naciones Unidas para el Medio Ambiente, Oficina Regional para América Latina y el Caribe/ Quercus Consultoría Ecológica.

De la Mora-De la Mora, G. (2015). Redes sociales y Áreas Naturales Protegidas en la Zona Metropolitana de Monterrey, Nuevo León. Economía, Sociedad y Territorio, XV(49). doi: http:// dx.doi.org/10.22136/est002015709

De la Mora-De la Mora, G. \& Montaño Salazar, R. (2016, marzo-agosto). ¿ Hacia la construcción de una gobernanza ambiental participativa? Estudio de caso en el Área Metropolitana de Guadalajara. Intersticios Sociales, 11(6), 1-27. Recuperado el 12 de noviembre de 2016, de http://www.intersticiossociales.com

De Sartre, X. A., Oszwald, J., Catro, M. \& Dufour, S. (2014). Political ecology des services écosystémiques. Bruselas: Peter Lang.

Dempsey, J. \& Robertson Morgan, M. (2012). Ecosystem services: Tensions, impurities and points of engagemente within neoliberalism. Progress in Humane Geography, 36(6), 758-779. doi: $10.1177 / 0309132512437076$ 
Díaz Vázquez, J. (2012). Servicios ecosistémicos culturales y de regulación en el Parque Bosque Colomos para el bienestar social. Tesis de Maestría en Ciencias de la Salud Ambiental. Centro Universitario de Ciencias Biológicas y Agropecuarias, Centro Universitario de Ciencias de la Salud. Universidad de Guadalajara. Jalisco, México.

Dos Santos, V. M. N. y De la Corte Bacci, D. (2017). Proposta para governala ambiental ante os dilemas socioambientais urbanos. Estudos Avancados, 31(89), 199-212. doi: 10.1590/ s0103-40142017.31890017

Elmqvist, T., Fragkias, M., Goodness, J., Güneralp, B., Marcotullio, P. J. \& McDonald, R. et al. (2013). Urbanization, Biodiversity and Ecosystem Services: Challenges and Opportunities. Dordrecht, The Netherlands: Springer Netherlands.

Ernstson, H., Sörlin, S. \& Elmqvist, T. (2008). Social movements and ecosystem services-the role of social network structure in protecting and managing urban green areas in Stockholm. Ecology and Society, 13(2). Recuperado el 13 de noviembre de 2016, de www. ecologyandsociety.org/vol13/iss2/art39/

Fernández Eguiarte, A., Uribe Cruz, F., Ramírez del Razo, I., Jesús Apolinar, B. de \& Vázquez Márquez, A. (2002). Evaluación del avance de la mancha urbana sobre el área natural protegida de la Cañada de los Dinamos. Gaceta Ecológica, (62), 56-67.

Fischer, J. A. \& Brown, K. (2014, diciembre). Ecosystem services concepts and approaches in conservation: Just a rhetorical tool? Ecological Economics, 108, 257-265. doi: http://dx.doi .org/10.1016/j.ecolecon.2014.11.004

Flores-Xolocotzi, R. \& González-Guillén, M. de J. (2010, enero-junio). Planificación de sistemas de áreas verdes y parques públicos. Revista Mexicana de Ciencias Forestales, 1(1), 17-24.

Fuentes, M. L. (2015, 1 de septiembre). México social: un país de 120 millones de habitantes. Excélsior. Consultado el 10 de octubre de 2016, de https://www.excelsior.com.mx/nacional /2015/09/01/1043298

Galicia, E. (2018). Vivir sin agua. Cienciorama. [Revista digital de divulgación científica]. Recuperado el 16 de julio de 2018, de http://www.cienciorama.unam.mx/\#!autor/102/efrain-s -galicia

García Barrios, R. (2014, septiembre). El origen de la reserva ecológica de la UNAM en CU: historia de un conflicto patrimonial y ambiental. Cultura y Representaciones Sociales, 9(17), 177-226.

Gómez-Baggethun, E., De Groot, R., Lomas, P. L. \& Montes, C. (2010, abril). The history of ecosystem services in economic theory and practice: From early notions to markets 
and payment schemes. Ecological Economics, 69(6), 1209-1218. doi: dx.doi.org/10.1016 /j.ecolecon.2009.11.007

Grimm, N. B., Grove, M. J., Pickett Steward, T. A. \& Redman, C. L. (2008). Integrated approaches to Long-Term Studies of Urban Ecological Systems. En J. M. Marzluff, E. Schulenberger, W. Endlicher, M. Alberti, G. Bradley, C. Ryan, C. ZumBrunnen \& U. Simon (Eds.), Urban Ecology. An international perspective on the interaction between humans and nature. Nueva York, Estados Unidos: Springer.

Hernández Rivera, M. G. \& Torres Hernández, L. (2015). Analysis of two protected areas in relation to the growth of the metropolitan area of Xalapa, Veracruz. Investigaciones Geográficas, (87), pp. 51-61. doi: http://dx.doi.org/10.14350/rig.39077

Holdgate, M., Kassas, M. \& White, G. (1982). World environmental trends between 1972 and 1982. Environmental Conservation, 9(1). doi: dx.doi.org/10.1017/S0376892900019408

Jujnovsky, J., Almeida-Leñero, L., Bojorge-García, M., Monges Yani, L., Cantoral Uriza, E. \& Mazari-Hiriarte, M. (2010). Servicios Ecosistémicos hidrológicos: calidad y cantidad del agua en el río Magdalena, Ciudad de México. Hidrobiológica, 20(2), 113-126.

Lascoumes, P. \& Le Gales, P. (2007, enero). Introduction: Understanding Public Policy through Its Instruments-From the Nature of Instruments to the Sociology of Public Policy Instrumentation. Governance: An International Journal of Policy, Administration, and Institutions, 20(1), 1-21.

Livesley, S. J., McPherson, E. G. \& Calfapietra, C. (2016). The Urban Forest and Ecosystem Services: Impacts on Urban Water, Heat, and Pollution Cycles at the Tree, Street, and City Scale. Journal of Environmental Quality, (45), 119-124. doi: 10.2134/jeq2015.11.0567

Mann, C. \& Simons, A. (2015, diciembre). Local emergence and international developments of conservation trading systems: Innovation dynamics and related problems. Environmental Conservation, 42(4), 325-334.

Marín Origel, Y., Orozco Hernández, M. \& Tapia Quevedo, J. (2013, mayo). Medio ambiente urbano en Huixquilucan, México. Urbano, 16(27), 41-47.

Marshall, F., Dolley, J., Bisht, R., Priya, R., Waldman, L., Amerasinghae, P. et al. (2018). Ecosystem services and poverty alleviation in urbanising contexts. En K. Schreckenberg, G. Mace \& M. Poudyal (Eds.), Ecosystem services and poverty alleviation. Trade-offs and governance (pp. 111-125). Nueva York: Routledge/Taylor and Francis.

G. De la Mora-De la Mora | Aproximación sociopolítica para el análisis de políticas de conservación en contextos urbanos: entre servicios ambientales y áreas naturales protegidas | Perfiles Latinoamericanos, 27(53) 
Mazari-Hiriart, M., Pérez-Ortiz, G., Orta-Ledesma, M. T., Armas-Vargas, F., Tapia Marco, A., Solano-Ortiz et al. (2014, julio). Final opportunity to rehabilitate an urban river as a water sources for Mexico City. PLOS ONE, 9(7). doi: dx.doi.org/10.1371/journal.pone.0102081

MEA. (2005b). Ecosystems and Human Well-Being. Synthesis. A report of the Millennium Ecosystem Assessment. En Millennium Ecosystem Assessment. Washington, D.C. Recuperado el 11 de mayo de 2016, de http://www.millenniumassessment.org/documents /document.356.aspx.pdf

Mekonnen Mesfin, M. \& Hoekstra Arjen, Y. (2016, febrero). Four billion people facing severe water scarcity. Science Advances, 2(2). doi: 10.1126/sciadv.1500323

Merlín-Uribe, Y., Contreras-Hernández, A., Astier-Calderón, M., Jensen, O., Zaragoza, R. \& Zambrano, L. (2013). Urban expansion into a protected natural area in Mexico City: Alternative management scenarios. Journal of Environmental Planning and Management, 56(3). doi: dx.doi.org/10.1080/09640568.2012.683686

Meuleman, L. (2010). The cultural dimension of metagovernance: Why governance doctrines may fail. Public Organization Review, 10(1), 49-70.

Nava-López, M. Z., Diemont Stewart, A. W., Hall, M. \& Ávila-Akerberg, V. (2016, junio). Riparian Buffer Zone and whole watershed influences on river water quality: Implications for ecosystem services near megacities. Environmental Processes, 3(2), 277-305. doi: 10.1007/ s40710-016-0145-3

Nelson, G. C. (2005). Drivers of ecosystem change: Summary chapter. En R. M. Hassan, R. Scholes, N. Ash \& R. E. Scholes, Ecosystem and Well-Being 1: Current State and Trends. Island Press.

Nordling, L. (2018). Ecologists up in arms over Cape Town's plans to ease water crisis by drilling into aquifer. Sciences. doi: 10.1126/science.aat 4351

Norgaard, R. B. (2013). The Econocene and the California Delta. San Francisco Estuary \& Watershed Science: A Broad Perspective, 11(3). doi: http://escholarship.org/uc/item 14h98t2m0\#page-1

Pagiola, S., Von Ritter, K. \& Bishop, J. (2004). How much is an ecosystem worth? Assessing the economic value of conservation. Washington, Estados Unidos: IUCN/TNC/WB.

Paré, L. \& Fuentes, T. (2007). Gobernanza ambiental y politicas públicas en Áreas Naturales Protegidas. Lecciones desde Los Tuxtlas. México: IIS, UNAM. 
Paré, L. \& Robles, C. (2008). Gestión territorial del agua en la subcuenca Texizapa-Huazuntlán, Sur de Veracruz. En L. Paré, D. Robinson \& M. A. González (Coords.), Gestión de Cuencas y Servicios Ambientales. Perspectivas Comunitarias (pp. 139-162). México: Semarnat/InECOL/ RAISES/PNUMA/Ítaca.

Paz Salinas, M.F. (2014). Conflictos socioambientales en México ¿Qué está en disputa? En M. F. Paz \& N. Risdell (Coords.), Conflictos, conflictividades y movilizaciones socioambientales en México: problemas comunes, lecturas diversas (pp. 13-58). México: unAm/M. A. Porrúa.

Perevochtchikova, M. \& Oggioni, J. (2014). Global and Mexican analytical review of the state of the art on ecosystem and environmental services: A geographical approach. Investigaciones Geográficas, (85), [Boletín], 47-65. doi: dx.doi.org/10.14350/rig.41239

Perevochtchikova, M. \& Vázquez Beltrán, A. (2012). The Federal Program of Payment for Hydrological Environmental Services as an alternative instrument for integrated wáter resources management in Mexico City. The Open Geography Journal, (5), 35-46.

Pérez-Campuzano, E., Avila-Foucat, S. \& Perevochtchikova, M. (2016, febrero). Environmental policies in the peri-urban área of Mexico City: The perceived effects of three environmental programs. Cities, 50, 129-136. doi: dx.doi.org/10.1016/j.cities.2015.08.013

Pimienta-Barrios, E., Robles-Murgía, C., Carvajal, S., Muñoz-Urias, A., Martínez-Chávez, C. \& León-Santos, S. de. (2014). Servicios ambientales de la vegetación en ecosistemas urbanos en el contexto del cambio climático. Revista Mexicana de Ciencias Forestales, 5(22), 28-39.

Ribeiro, F. P. \& Ribeiro, K.T. (2016, julio). Participative mapping of cultural ecosystem services in Pedra Branca State Park, Brazil. Natureza \& Conservação, 14(2), 120-127. doi: https:// doi.org/10.1016/j.ncon.2016.09.004

Rival, L. \& Muradian, R. (2013). Introduction: Governing the provision of ecosystem services. En M. Roldan \& L. Rival (Eds.), Governing the provision of ecosystem services (pp. 1-17 ). Dordrecht: Springer.

Romero, H., Vásquez, A., Fuentes, C., Salgado, M., Schmidt, A. \& Banzhaf, E. (2012). Assessing urban environmental segregation (UES). The case of Santiago de Chile. Ecological Indicators, 23, 76-87. doi: 10.1016/j.ecolind.2012.03.012

Schröter, M., Van der Zanden, E. H., Van Oudenhoven, A. P. E., Remme, R. P., Serna-Chavez, H. M. \& De Groot et al. (2014). Ecosystem Services as a contested concept: A synthesis of critique and counter-arguments. Conservation Letters, 7(6), 514-523. 
Schteingart, M. R. (2006, octubre). Migraciones, expansión urbana e impacto ambiental en la región metropolitana de la Ciudad de México. Ponencia presentada en el II Simposio Internacional. Desertificación y Migraciones. Universidad de Almería, España.

Silke, C., Cotler, H., Morales, L.M., Sommer, Irene \& Carmona, E., (2008). Identificación de los servicios ambientales potenciales de los suelos en el paisaje urbano del Distrito Federal. Investigaciones Geográficas, 66, 81-104.

Simonian, L. (1999). La defensa de la tierra del Jaguar. Una historia de la conservación en México. México: Semarnap/Instituto de Ecología/Conabio.

Tlapa Almonte, M. (2011). Áreas Naturales Protegidas periurbanas del Área Metropolitana del Estado de Puebla. Tesis para obtener el grado de Doctora en Ciencias. Postgrado en Estrategias para el Desarrollo Agrícola Regional. Colegio de Postgraduados, Campus Puebla. México. Recuperado de http://hdl.handle.net/10521/564

Torres, J. M. (2010). ProÁrbol, un programa para restaurar, manejar y conservar bosques. En J. Carabias, J. Sarukhán, J. de la Maza \& C. Galindo (Coords.), Patrimonio natural de México. Cien casos de éxito (pp. 102-103). México: Conabio/Semarnat.

Tress G., Tress B. \& Fry, G. (2004). Clarifying integrative research concepts in landscape ecology. Landscape Ecology, (20), 479-493.

Van den Broeck, P. (2008). The Changing Position of Strategic Spatial Planning in Flanders. A Socio-Political and Instrument-Based Perspective. International Planning Studies, 13(3), 261-283. doi: 10.1080/13563470802521457

Wallace, K. J. (2007, octubre). Classification of ecosystem services: Problems and solutions. Biological Conservation, 139(3-4), 235-246. doi: http://dx.doi.org/10.1016/j.biocon.2007 .07 .015

West, P., Igoe, J. \& Brockington, Dan. (2006, junio). Parks and Peoples: The social impact of protected áreas. Annual Review of Anthtropology, 5(35), 251-277.

Wunder, S. (2005a). Payments for environmental services: Some nuts and bolts. Cifor. Occasional Paper, 42(24). Recuperado el 1 de enero de 2017, de http://www.cifor.org/publications/ pdf_files/OccPapers/OP-42.pdf

Wunder, S., Dung, B. \& Ibarra, E. (2005). Payment is good, control is better: Why payments for forest environmental services in Vietnam have so far remained incipient. Indonesia: CIFOR Editors. 